\title{
CONSUMO DE FRUTAS EM UNIDADE DE ALIMENTAÇÃO E NUTRIÇÃO NO MUNICÍPIO DE SÃO PAULO: UM ESTUDO DE CASO
}

\section{CONSUMPTIN OF FRUIT AT A FOOD SERVICE UNIT IN THE CITY OF SÃO PAULO: A CASE STUDY}

\author{
Stephanie Bento da Silva ${ }^{1}$ \\ Mônica Gloria Neumann Spinelli²
}

Resumo: Evidências científicas revelam que frutas, legumes e verduras são alimentos indispensáveis para manter uma boa saúde, pois estes auxiliam na prevenção das doenças cardiovasculares e vários tipos de câncer. Entretanto, a Pesquisa de Orçamento Familiar POF - 2008/2009 revelou que o consumo de frutas e hortaliças pelos brasileiros representa, aproximadamente, um quarto da quantidade recomendada. Com o objetivo de se avaliar o consumo de frutas, observou-se durante as refeições desjejum e almoço as escolhas feitas pelos clientes de um restaurante de empresa no município de São Paulo. De acordo com os resultados obtidos, verificou-se que, no desjejum, clientes de trabalho operacional não têm o hábito de consumir frutas, enquanto que os funcionários administrativos sempre optam por estas. No almoço, independentemente do tipo de atividade, ambos preferem consumir doces. Diante do exposto, atividades de educação nutricional devem ser realizadas com o intuito de informar sobre os benefícios do consumo desse grupo de alimentos e melhorar o hábito nutricional dos trabalhadores dessa empresa.

Palavras-chave: consumo frutas; preferências alimentares; educação nutricional.

\begin{abstract}
Scientific evidence shows that fruit and vegetables are necessary to maintain good health and to prevent cardiovascular diseases and cancers. However, the research "Pesquisa de Orçamento Familiar POF 2008/2009" revealed that Brazilians' consumption of fruits and vegetables represents approximately a quarter of the recommended amount. In order to evaluate fruit consumption, workers' choices were observed during the breakfast and lunch in a food service in the city of São Paulo. According to the results, operational workers do not have the habit of consuming fruits at breakfast, while administrative staff always takes this option. At lunch, both kinds of workers prefer to consume dessert instead of fruit. Thus, nutrition education activities should be undertaken in order to inform workers about the benefits of consuming this food group and improve the nutritional habits of the employees of this company.
\end{abstract}

Keywords: fruit consumption; food preferences; nutrition education.

\footnotetext{
${ }^{1}$ Graduada em Nutrição - Universidade Paulista - UNIP, Brasil. E-mail: stephaniebento.nutri@gmail.com.

2 Doutora em Saúde Pública (área de concentração - Nutrição) - Universidade de São Paulo - USP. Docente da UNIP, Brasil. E-mail: monicaspi404@gmail.com.
} 


\section{INTRODUÇÂO}

Nos últimos anos, o Brasil tem passado por uma intensa transição nutricional, gerando aumento da obesidade e doenças crônicas não transmissíveis, como diabetes mellitus, câncer e doenças cardiovasculares (MATTOS; NEVES, 2009).

Várias recomendações têm sido feitas com relação às frutas. O Programa de Alimentação do Trabalhador (PAT) prevê a oferta de frutas em todas as refeições (BRASIL, 2006a). O Guia Alimentar para a População Brasileira, de 2006, recomenda o consumo de, pelo menos, três porções ao dia, e o Guia Alimentar para a População Brasileira, de 2014, apesar de não recomendar especificamente o número de porções, ressalta a importância das frutas para a saúde, além de orientar a preferência que deve ser dada aos alimentos e não às preparações (BRASIL, 2006b; BRASIL, 2014).

As frutas são indispensáveis ao bom funcionamento do organismo. Os nutrientes mais comuns nas frutas são vitaminas, carboidratos, proteínas, fibras, minerais solúveis e insolúveis e água (ABREU; SPINELLI, 2014). Possuem carboidratos complexos, com fibra natural intacta, que têm distintas vantagens sobre os carboidratos simples, tais como menor índice glicêmico, maior saciedade e propriedades de ligação com o colesterol. As fibras alimentares, principalmente as solúveis, possuem efeito benéfico, reduzindo os níveis de colesterol total e LDL, e melhorando a tolerância à glicose. Além disso, nas frutas e hortaliças também são encontradas substâncias antioxidantes, como vitamina E, pigmentos carotenóides, vitamina C, flavonóides e outros compostos fenólicos, que têm sido associadas a tais benefícios (FRANZ et al., 2003; LOCK et al., 2005).

Há evidências científicas de que frutas, legumes e verduras são alimentos indispensáveis para manter uma boa saúde, pois auxiliam na prevenção das doenças cardiovasculares e vários tipos de câncer. Acredita-se que mais de 2,7 milhões de vidas poderiam ser potencialmente salvas todos os anos, se houvesse maior consumo de frutas, legumes e verduras (WHO/FAO, 2003).

A Pesquisa de Orçamento Familiar POF - 2008/2009 revelou que o consumo de frutas e hortaliças foi de $2,8 \%$ do total de calorias dos alimentos disponíveis para consumo nos domicílios dos brasileiros, o que representa, aproximadamente, um quarto da quantidade recomendada (IBGE, 2010).

Mesmo sendo reconhecido o papel fundamental que as frutas exercem para a saúde humana, em especial, como fator de proteção contra as doenças crônicas não transmissíveis, o consumo desse alimento é considerado baixo em vários países (OLIVEIRA et al., 2008).

Cada vez mais as empresas se conscientizam de que, para ter competitividade, é preciso investir em programas de qualidade de vida para seus funcionários e a alimentação tem um papel importante nesse processo. Para tanto, programas de educação alimentar visam adotar um processo educativo permanente, fazendo do ato de se alimentar no ambiente de trabalho uma fonte de saúde, bem estar, qualidade de vida e de produtividade 
dos seus funcionários (BRASIL, 2001).

Tem-se observado que apenas a informação sobre os benefícios do consumo de frutas para a saúde não tem provocado mudanças nos hábitos alimentares dos indivíduos. Dessa forma, as estratégias ambientais têm recebido maior atenção, uma vez que elas agem para adotar uma alimentação saudável, ou seja, o ambiente de trabalho tem sido reconhecido como um lugar estratégico para a promoção da saúde e de uma alimentação saudável (OLIVEIRA et al., 2008).

O Programa de Alimentação do Trabalhador (PAT) recomenda que os cardápios ofereçam, no mínimo, uma porção de frutas nas refeições principais (almoço, jantar e ceia) e pelo menos uma porção nas refeições menores (café da manhã e lanche) (BRASIL, 2006a).

Assim, o PAT pode ser considerado um elemento importante na promoção de alimentação saudável para a população trabalhadora. As empresas filiadas ao PAT oferecem uma alimentação adequada, mas é necessário que o trabalhador saiba escolher bem os alimentos que Ihes são ofertados, para que a sua alimentação seja realmente saudável (SOUZA; SILVA, 2010).

Diante do exposto, este estudo teve como objetivo avaliar o consumo de frutas nas refeições, pelos trabalhadores de uma empresa no município de São Paulo.

\section{METODOLOGIA}

O presente estudo transversal observacional envolveu clientes de uma empresa localizada na cidade de São Paulo, inscrita no PAT. A gestão da Unidade de Alimentação e Nutrição (UAN) é terceirizada.

O sistema de distribuição na unidade é centralizado, sendo as frutas distribuídas, no mesmo local, por meio de autosserviço ou self-service, sistema em que cada indivíduo tem o livre arbítrio de fazer suas escolhas, seja pela fruta, sobremesa doce ou os dois.

Foi avaliado o consumo de frutas, durante duas semanas, por clientes de trabalho administrativo e, em outras duas, pelos de trabalho operacional.

Os dados foram registrados em uma ficha elaborada pela pesquisadora:

Para o desjejum utilizou-se o seguinte critério:

- Escolha de fruta; e

- Sem escolha de fruta

Para o almoço:

- Escolha de fruta;

- Sem escolha de fruta; 
- Escolha de fruta e doce; e

- Escolha apenas de doce.

A avaliação foi realizada nos horários de maior movimento de clientes (desjejum: 07h00 às 8h30; almoço: $11 \mathrm{~h} 30$ às 12h30), em dias não consecutivos, e, assim, a escolha de cada cliente foi registrada na ficha.

Como medida ética, para a concretização deste estudo, foi solicitada à nutricionista da UAN a permissão para a realização do estudo, garantindo o sigilo sobre a identidade do local.

\section{RESULTADOS}

$\mathrm{Na}$ UAN são oferecidas frutas no desjejum, e no almoço a sobremesa é composta por frutas e doces. O método de distribuição das refeições é self-service com livre demanda, ou seja, o funcionário tem a liberdade de escolher fruta, doce, as duas opções ou nenhuma delas.

De acordo com as escolhas feitas, pôde-se observar que houve diferenças de consumo, em relação aos clientes, segundo trabalho administrativo e operacional.

\section{Gráfico 1 - Consumo de frutas no desjejum dos clientes de trabalho administrativo. \\ UAN, São Paulo, 2014}

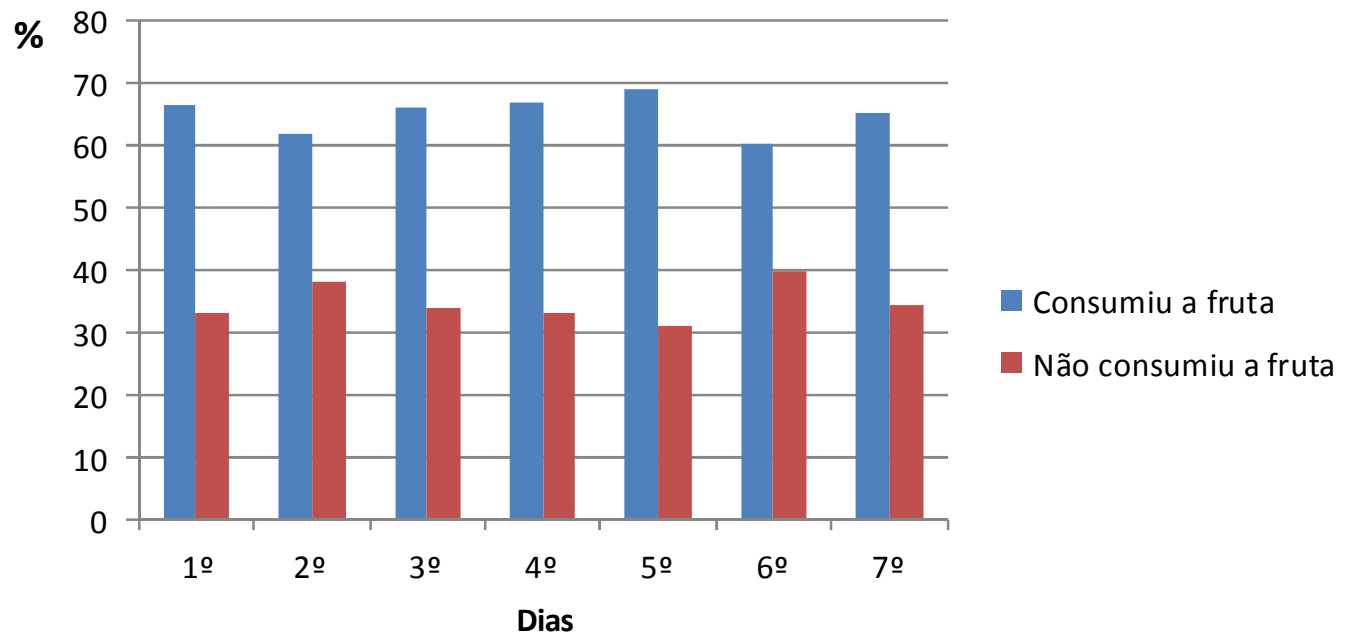


Gráfico 2 - Consumo de frutas no desjejum dos clientes de trabalho operacional. UAN, São Paulo, 2014

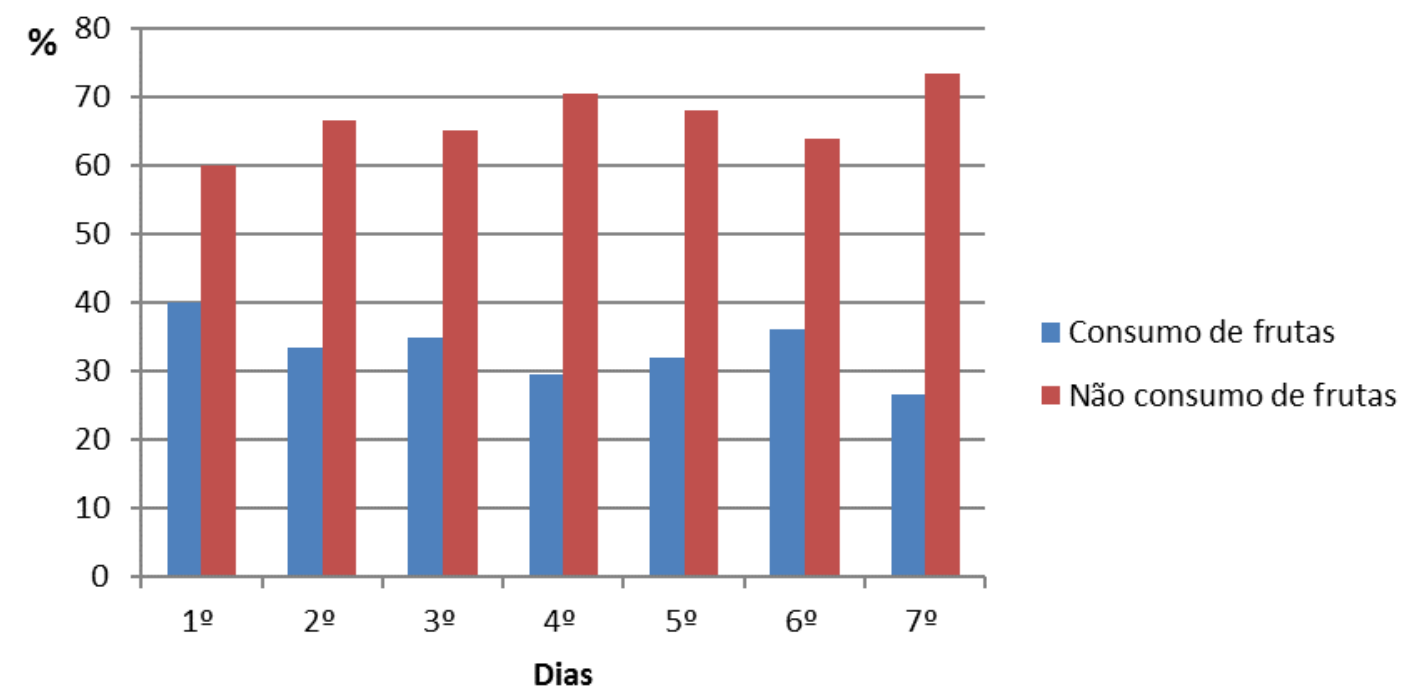

Tabela 1 - Escolha de frutas e sobremesas no almoço em refeitório dos clientes que exercem trabalho administrativo. UAN, São Paulo, 2014

\begin{tabular}{|c|c|c|c|c|c|c|c|c|c|}
\hline \multirow{2}{*}{$\begin{array}{l}\text { Dia } \\
\text { avaliado }\end{array}$} & \multirow[t]{2}{*}{$\begin{array}{c}\text { № } \\
\text { clientes }\end{array}$} & \multicolumn{2}{|c|}{ Apenas fruta } & \multicolumn{2}{|c|}{$\begin{array}{c}\text { Sem } \\
\text { sobremesa }\end{array}$} & \multicolumn{2}{|c|}{ Fruta + doce } & \multicolumn{2}{|c|}{ Somente doce } \\
\hline & & $\mathbf{n}$ & $\%$ & $\mathbf{n}$ & $\%$ & $\mathbf{n}$ & $\%$ & $\mathbf{n}$ & $\%$ \\
\hline 1 & 171 & 37 & 21,6 & 38 & 22,3 & 26 & 15,2 & 70 & 40,9 \\
\hline 2 & 129 & 6 & 3,7 & 1 & 0,6 & 26 & 16,0 & 129 & 79,7 \\
\hline 3 & 113 & 27 & 23,9 & 18 & 15,9 & 23 & 20,3 & 45 & 39,9 \\
\hline 4 & 167 & 33 & 19,8 & 58 & 34,7 & 15 & 8,9 & 61 & 36,6 \\
\hline 5 & 144 & 11 & 7,6 & 33 & 22,9 & 26 & 18,0 & 74 & 51,5 \\
\hline 6 & 160 & 35 & 21,9 & 46 & 28,7 & 23 & 14,4 & 56 & 35,0 \\
\hline 7 & 162 & 17 & 10,5 & 25 & 15,4 & 45 & 27,8 & 75 & 46,3 \\
\hline $\bar{x}$ & 149,4 & 23,7 & 15,6 & 31,28 & 20,1 & 26,3 & 17,2 & 72,8 & 47,1 \\
\hline DP & & 12,4 & & 18,75 & & 9,1 & & 27,0 & 12,4 \\
\hline
\end{tabular}


Tabela 2 - Escolha de frutas e sobremesas no almoço em refeitório dos clientes que exercem trabalho operacional. UAN, São Paulo, 2014

\begin{tabular}{|c|c|c|c|c|c|c|c|c|c|}
\hline \multirow{2}{*}{$\begin{array}{l}\text { Dia } \\
\text { avaliado }\end{array}$} & \multirow{2}{*}{$\begin{array}{l}\text { № } \\
\text { clientes }\end{array}$} & \multicolumn{2}{|c|}{ Apenas fruta } & \multicolumn{2}{|c|}{$\begin{array}{c}\text { Sem } \\
\text { sobremesa }\end{array}$} & \multicolumn{2}{|c|}{ Fruta + doce } & \multicolumn{2}{|c|}{ Somente doce } \\
\hline & & $\mathbf{n}$ & $\%$ & $\mathbf{n}$ & $\%$ & $\mathbf{n}$ & $\%$ & $\mathbf{n}$ & $\%$ \\
\hline 1 & 54 & 4 & 7,4 & 0 & 0 & 19 & 35,2 & 31 & 57,4 \\
\hline 2 & 57 & 9 & 15,7 & 13 & 22,8 & 17 & 29,8 & 18 & 31,6 \\
\hline 3 & 44 & 4 & 9,1 & 9 & 20,4 & 10 & 22,7 & 21 & 47,8 \\
\hline 4 & 61 & 12 & 19,7 & 12 & 19,7 & 14 & 22,9 & 23 & 37,7 \\
\hline 5 & 38 & 2 & 5,3 & 1 & 2,6 & 14 & 36,8 & 21 & 55,3 \\
\hline 6 & 31 & 4 & 12,9 & 11 & 35,5 & 7 & 22,6 & 9 & 29,0 \\
\hline 7 & 31 & 10 & 32,2 & 7 & 22,6 & 7 & 22,6 & 7 & 22,6 \\
\hline $\bar{x}$ & 45,1 & 6,4 & 14,6 & 7,6 & 17,7 & 12,6 & 27,5 & 18,6 & 40,2 \\
\hline DP & & 3,8 & & 5,2 & & 4,7 & & 8,3 & 3,8 \\
\hline
\end{tabular}

Observa-se no Gráfico 1 que o hábito de se servir de frutas no desjejum em todos os dias ficou superior a $60 \%$, com média de $65,2 \%$, para os clientes que exercem trabalho administrativo, ou seja, mais da metade dos trabalhadores administrativos consomem frutas nessa refeição, enquanto os que exercem trabalho operacional (Gráfico 2) não têm esse hábito, com uma escolha inferior a $40 \%$ e média de $33,2 \%$. Os que não se serviram ficam em torno de 60 e 74\%. Os valores obtidos no desjejum dos que exercem trabalho operacional são inversos aos dos que têm trabalho administrativo.

Comparando as médias entre almoço dos dois tipos de clientes, conclui-se que ambos preferem o consumo de doces ao invés de frutas, uma vez que, em média, $47,1 \%$ dos administrativos e $40,2 \%$ dos operacionais se servem apenas de doce, ou seja, quase metade dos comensais não se serve de fruta.

Nota-se que os clientes de trabalho administrativo têm um maior consumo quando comparado ao dos clientes que exercem trabalhos operacionais, embora ambos optem, na maioria das vezes, pela sobremesa doce ao invés de frutas.

\section{DISCUSSÃO}

O ambiente de trabalho é um local em que as pessoas passam cerca de $1 / 3$ do dia. Além disso, é ambiente propício para troca de informações, espaço de relações sociais e em que muitos fazem duas das três principais refeições diárias. Dessa forma, é um lugar no qual os fatores ambientais, organizacionais e pessoais interagem, interferindo na saúde e no bem estar das pessoas (OLIVEIRA et al., 2008). 
Observa-se, neste estudo, que clientes que exercem trabalho operacional, que na maioria das vezes apresentam baixa escolaridade e recebem salários menores, têm um menor consumo de frutas no desjejum, se comparados aos clientes de função administrativa. No trabalho feito por Oliveira et al. (2008), foi relatado que $55,7 \%$ da população estudada consumiu frutas todos os dias, porém observou-se que trabalhadores com renda inferior a 20 salários mínimos apresentaram seis vezes menos chances do que os demais de consumirem frutas todos os dias, coincidindo com os resultados evidenciados no presente estudo.

Verificou-se, neste trabalho, que, no almoço, 40,2\% dos clientes que exercem trabalho operacional e $47,1 \%$ dos que executam trabalho administrativo optaram por doce como sobremesa ao invés das frutas. Esse fato corrobora com os resultados encontrados em pesquisa realizada por Figueiredo, Jaime e Monteiro (2008), que demonstrou baixa frequência do consumo de frutas na população que reside em São Paulo e com os de Höfelmann, Rieckes e Azevedo (2005), que relatam expressiva porcentagem de consumo insuficiente de frutas entre colaboradores de uma UAN em Santa Catarina.

Fonseca et al. (2012), em restaurante universitário no Maranhão, encontraram resultado diverso ao do presente estudo. Dos 130 clientes avaliados, 86,2\% afirmaram preferir frutas ao invés de sobremesas doces na refeição.

Outro fato identificado no presente estudo foi a falta de orientação e educação nutricional aos clientes, contrária à política do PAT. Esta é importante para conscientizá-los sobre os benefícios do consumo adequado de frutas para a saúde, o que poderia contribuir para o aumento da escolha e consumo destas, que lhes são ofertadas nas refeições da empresa pesquisada. Embora o objetivo da UAN seja fornecer refeições equilibradas nutricionalmente, parece haver maior valorização do atendimento aos desejos sensoriais e hedônicos dos usuários, como demonstram Höffelmann, Rieckes e Azevedo (2005) que evidenciaram alto consumo de gorduras e consumo insuficiente de frutas; e Höfelmann e Garcia (2005), mostrando que de 75 clientes que consumiram sobremesa, apenas 16\% solicitaram frutas in natura.

Franco (2014) demonstrou, em seu estudo, baixo consumo de frutas e hortaliças pelos clientes de uma empresa. Após intervenção nutricional, na qual foram realizadas atividades presenciais com distribuição de materiais educativos e ações de comunicação, houve aumento de $38 \%$ no consumo de frutas e hortaliças no almoço dos trabalhadores, o que evidencia que atividades de educação nutricional podem contribuir para mudança e adequação de hábitos alimentares dos indivíduos.

Lipi e Santos (2007), após realizarem intervenção nutricional em uma UAN, verificaram que os resultados da primeira semana, em que $88 \%$ dos usuários optaram por consumo de doces e $12 \%$ por fruta, passaram, após três semanas de intervenção, para $68 \%$ e $32 \%$, respectivamente, mostrando que a educação nutricional deve ser incorporada de forma contínua às rotinas das UANs, para que haja melhora no hábito alimentar das pessoas. 
Dessa forma, tanto a oferta de frutas nas refeições fornecidas pela empresa, quanto as informações e ambientes temáticos, que favoreçam escolhas mais saudáveis, são pontos importantes para a promoção da saúde do trabalhador (OLIVEIRA et al., 2008).

\section{CONCLUSÃO}

Conclui-se que, embora o PAT preconize a oferta diária de frutas em todas as refeições das empresas a ele filiadas, somente o desenvolvimento do programa e incorporação das frutas no cardápio não torna essa condição suficiente para alterar os comportamentos alimentares a ponto de contribuir na melhoria do estado nutricional, saúde e qualidade de vida dos trabalhadores.

Assim, os processos educativos em alimentação e nutrição não devem ser apenas uma recomendação do programa. É necessário que sejam estimulados, no ambiente de trabalho, por meio de divulgação escrita no local das refeições, pela intranet da empresa, campanhas de conscientização e pela própria apresentação do alimento durante a refeição, como apresentação de tipos de cortes diferentes, disposição estratégica no balcão de distribuição e variedades.

É importante que se monitore o consumo e se façam novos estudos sobre o tema, procurando maior compreensão sobre a formação e interferência do hábito alimentar e de mudanças nas atitudes.

Deve-se compreender o espaço de trabalho como local de implantação de ações fomentadoras de mudanças comportamentais em grande escala. Cabe ao nutricionista, dentro de suas atribuições específicas, posicionar-se como agente de transformações, fomentando ações de educação e/ou reeducação alimentar e nutricional.

A empresa deve levar em conta que a melhora do hábito nutricional do trabalhador, reflete em seu estado de saúde e, consequentemente, no seu desempenho no trabalho e na redução do absenteísmo.

\section{REFERÊNCIAS}

ABREU, E. S.; SPINELLI, M. G. N. Seleção e Preparo de Alimentos: gastronomia e nutrição. São Paulo: Metha, 2014. 411p.

BRASIL. Ministério do Trabalho e Emprego. Portaria Interministerial n. 66, de 25 de agosto de 2006. Altera os parâmetros nutricionais do Programa de Alimentação do Trabalhador PAT. Diário Oficial da União, Brasília, 28 ago. 2006a. Disponível em: <http://www3.mte.gov.br/Empregador/PAT/Legislacao/Conteudo/port66.pdf>. Acesso em: 18 jun. 2015. 
. Ministério do Trabalho e Emprego. Orientação da Educação Alimentar. Brasília: MTE, SIT, DSST, 2001. Disponível em: $<$ http://portal.mte.gov.br/data/files/FF80808144C1D57 $\quad$ F0144E 648 E9A 50 EA8/Manual\%200rienta\%C3\%A7\%C3\%A30\%20da\% 20Educa $\%$ CC3\%A7 $\quad \%$ C3 $\quad \%$ A30\%20Alimentar.pdf>. Acesso em: 18 jun. 2015.

Ministério da Saúde. Guia Alimentar para a População Brasileira, 2014. Disponível em: $<$ http://acesso.mte.gov.br/data/files/FF8080814B74A055014BB67117AC36E8/quia alimentar populacao brasileira.pdf >. Acesso em: 18 jun. 2015.

FIGUEIREDO, I. C. R.; JAIME, P. C.; MONTEIRO, C. A. Fatores associados ao consumo de frutas, legumes e verduras em adultos da cidade de São Paulo. Rev. Saúde Pública, São Paulo, v. 42, n. 5, p. 777-785, 2008.

FONSECA, J. M. et al. Avaliação da preferência de sobremesas fornecidas no restaurante universitário da Universidade Federal do Maranhão. In: REUNIÃO ANUAL DA SBPC, 64., 2012, Maranhão. Anais... São Luis: UFMA, 2012. Disponível em: $<$ www.sbpcnet.org.br/livro/64ra/resumos/resumos/6735.htm>. Acesso em 28 jul. 2014.

FRANCO, A. Avaliação do impacto de ações para promoção do consumo de frutas e hortaliças no ambiente de trabalho. DEMETRA: Alimentação, Nutrição \& Saúde, Rio de Janeiro, v. 9, n. 1, p. 185-186, 2014.

FRANZ, M. J. et al. Evidence-based nutrition principles and recommendations for the treatment and prevention of diabetes and related complications. Diabetes Care, Alexandria, v. 26, n. 1, p. S51-S61, 2003.

HÖFELMANN, D. A.; GARCIA, L. Análise das preferências alimentares dos clientes de um restaurante de culinária internacional a la carte. Nutr. Pauta, v. 13, n. 71, p. 18-24, 2005.

HÖFELMANN, D. A.; RIECKES, B. H.; AZEVEDO, L. C. Hábito alimentar e estado nutricional: fatores de risco cardiovascular entre colaboradores internos de uma Unidade de Alimentação e Nutrição. Nutr. Pauta, v. 13, n. 70, p. 42-45, 2005.

INSTITUTO BRASILEIRO DE GEOGRAFIA E ESTATíSTICA. Pesquisa de orçamentos familiares 2008-2009: avaliação nutricional da disponibilidade domiciliar de alimentos no Brasil. Rio de Janeiro: IBGE, 2010.

LIPI, M.; SANTOS, V. F. Avaliação de intervenção educativa na aceitação de frutas como sobremesa em uma unidade de alimentação e nutrição. Nutrire, v. 32, supl., p. 304-304, 2007.

LOCK, K. et al. The global burden of disease attributable to low consumption of fruit and vegetables: implications for the global strategy on diet. Bull World Health Organ. v. 83, n. 2, p. 100-108, 2005. 
MATTOS, P. F.; NEVES, A. S. A Importância da Atuação do Nutricionista na Atenção Básica à Saúde. Rev. Práxis, Fortaleza, v.1, n. 2, p. 11-15, 2009.

OLIVEIRA, S. P. et al. Promoção do consumo de frutas, legumes e verduras no ambiente de trabalho: diagnóstico inicial. 1. ed. Rio de janeiro: Embrapa Agroindústria de Alimentos. 2008.

SOUZA, C. E.; SILVA, A. B. G. Consumo alimentar habitual dos trabalhadores de uma empresa do Vale do Taquari - RS. Revista Destaques Acadêmicos, v. 2, n. 3, p. 19-25, 2010.

WHO. FAO. Diet, Nutrition and the Prevention of Chronic Diseases: report of a joint WHO/FAO expert consultation. Geneva: WHO, 2003. (WHO technical report series, 916). Disponível em: <http://www.fao.org/docrep/005/ac911e/ac911e00.HTM>. Acesso em: 12 abr. 2014. 